\title{
Effects of dapagliflozin on serum uric acid levels in hospitalized type 2 diabetic patients with inadequate glycemic control: a randomized controlled trial
}

This article was published in the following Dove Press journal:

Therapeutics and Clinical Risk Management

\section{Zhaohu Hao, ${ }^{1} *$ Xiao Huang, 2,* Hailin Shao,' Fengshi Tian ${ }^{1,3}$ \\ 'Department of Metabolic Disease Management Center, Tianjin 4th Central Hospital, Tianjin, China; ${ }^{2} \mathrm{NHC}$ Key Laboratory of Hormones and Development (Tianjin Medical University), Tianjin Key Laboratory of Metabolic Diseases, Tianjin Medical University Metabolic Diseases Hospital and Tianjin Institute of Endocrinology, Tianjin Medical University, Tianjin, China; ${ }^{3}$ Department of Cardiology, Tianjin 4th Central Hospital, The 4th Central Hospital Affiliated to Nankai University, The 4th Center Clinical College of Tianjin Medical University, Tianjin, China}

*These authors contributed equally to this work

Correspondence: Hailin Shao Department of Metabolic Disease Management Center, Tianjin 4th Central Hospital, No I, Zhongshan Road, Tianjin 300 I40, China

Tel +8622 26249403

Email keddyhm@163.com

Fengshi Tian Department of Cardiology, Tianjin 4th Central Hospital, Tianjin 4th Central Hospital Affiliated to Nankai University and The 4th Center Clinical College of Tianjin Medical University, No.I Zhongshan Road, Tianjin 300I40, China Tel +8622 2624928 I

Email fengshitian080I0II@I26.com
Background: Raised serum uric acid (SUA) level is commonly observed in patients with type 2 diabetes mellitus (T2DM) and is associated with increased morbidity and mortality. Sodium-glucose cotransporter 2 inhibitor, a novel oral diabetic drug, might exert a potential hypouricemic effect. We evaluated the effects of dapagliflozin on SUA levels in hospitalized T2DM patients with inadequate glycemic control.

Methods: In this randomized controlled trial, 59 T2DM hospitalized patients with inadequate glycemic control were assigned to the dapagliflozin $10 \mathrm{mg}$ group $(\mathrm{n}=29)$ or the control group $(n=30)$. The primary outcome was changes in SUA levels from the baseline to good glycemic control. Additional outcomes included correlations between baseline SUA levels, urinary parameters, and the changes in SUA levels. This trial is registered in the Chinese Clinical Trial Registry (number ChiCTR1800015830).

Results: Compared to baseline level, SUA levels had significantly decreased in both groups ( $P<0.001$ for the dapagliflozin group and $P=0.013$ for the control group). Mean changes from baseline in SUA levels for dapagliflozin vs the control group were 68.03 vs $25.90 \mu \mathrm{mol} / \mathrm{L}$ $(P=0.0406)$. Adjusted mean SUA levels were lower in the dapagliflozin group (273.28 vs $307.57 \mu \mathrm{mol} / \mathrm{L} ; P=0.0089)$. In T2DM patients treated with dapagliflozin, the decrease in SUA levels was positively correlated with baseline SUA levels $(P<0.0001)$ but not correlated with changes in 24-hour urine volume, 24-hour urine glucose, or 24-hour urinary uric acid.

Conclusion: Dapagliflozin could improve glycemic control and lower SUA levels in hospitalized patients with uncontrolled T2DM. Longer-time trials are required to further demonstrate the hypouricemic effect of dapagliflozin and explore the potential underlying mechanisms.

Keywords: serum uric acid, dapagliflozin, type 2 diabetes mellitus, 24-hour urinary uric acid, SGLT2 inhibitor

\section{Introduction}

Uric acid is the end product of purine metabolism, which has dual characteristics of antioxidant activity and promoting oxidation. Imbalance in uric acid production and excretion could cause hyperuricemia, which is considered to be a well-established risk factor for gout, nephropathy, and nephrolithiasis. ${ }^{1,2}$ Recently, hyperuricemia has been reported to be related to other conditions including atherosclerosis, hypertension, and cardiovascular diseases. ${ }^{3-6}$ Hyperuricemia and type 2 diabetes mellitus (T2DM) are closely interrelated. On the one hand, higher serum uric acid (SUA) may increase the development of insulin resistance, impaired glucose tolerance, and T2DM..$^{711}$ 
A previous study including 17,044 participants from China showed that increased uric acid level was associated with T2DM after adjusting for potential confounders. ${ }^{7}$ Another 15-year follow-up study suggested that compared with normal levels of SUA, hyperuricemia was independently related to 1.25 times of prediabetes, 1.36 times of insulin resistance, and 1.87 times of T2DM. ${ }^{10}$ On the other hand, renal dysfunction and hyperproduction of uric acid linked to hyperinsulinemia and insulin resistance in T2DM patients influenced the presence of hyperuricemia. ${ }^{12}$ In addition, hyperuricemia has been shown to be a risk factor for the progression of diabetic peripheral neuropathy, increased mortality rate, cancer incidence, atrial fibrillation, and metabolic syndrome in patients diagnosed with T2DM. ${ }^{13-16}$ Given the above adverse effects of hyperuricemia, it was proposed that lowering SUA level may benefit T2DM patients.

Sodium-glucose cotransporter 2 (SGLT2) inhibitor, a novel oral diabetic drug, can reduce blood glucose and improve glycemic control through an insulin-independent mechanism, and has been reported to be associated with a hypouricemic effect in patients with T2DM. ${ }^{17}$ Dapagliflozin was the first approved SGLT2 inhibitor for T2DM. ${ }^{18}$ Apart from glycemic control, beneficial effects including lowering of body weight, blood pressure reduction, and renal function protection have also been observed in T2DM patients taking dapagliflozin. ${ }^{19-22}$ In particular, dapagliflozin was reported to lower SUA levels in several studies. ${ }^{21,23-28}$ A review by Ahmadieh and Azar suggested a beneficial effect on uric acid levels following treatment by dapagliflozin. ${ }^{29}$ Notably, a recent meta-analysis of randomized control trials (RCTs) found a dose-dependent mode of dapagliflozin-induced hypouricemic effect from 5 to $50 \mathrm{mg}$ and the combined effect of reduction was 36.99 (95\% CI: $32.25-41.73) \mu \mathrm{mol} / \mathrm{L} .{ }^{30}$

However, none of the studies included in the metaanalysis were specially designed to assess the uric acidlowering effect of dapagliflozin. This study aims to evaluate the effect of dapagliflozin on SUA levels in hospitalized T2DM with inadequate glycemic control and to explore whether the potential underlying mechanisms were linked to baseline SUA levels and urinary parameters.

\section{Methods}

\section{Study eligibility criteria}

We recruited subjects with a primary diagnosis of T2DM aged $\geq 18$ years from January 2018 to May 2018 in the endocrinology department of Tianjin 4th Center Hospital. Patients had to have adhered to their previous hypoglycemic programs for at least 3 months prior to hospitalization with inadequate glycemic control (glycosylated hemoglobin, HbA1c $\geq 8 \%$ ).
Patients should have abstained from alcohol for 1 week before hospitalization to the end of the study. Drugs such as aspirin, thiazide diuretics, fructose, niacin, vitamin $\mathrm{C}$, atorvastatin, glucocorticoids, phenyl bromide malone, sodium bicarbonate, losartan, fenofibrate, reserpine, and amlodipine besylate that affected uric acid metabolism were prohibited.

Exclusion criteria were as follows: type 1 diabetes and other unusual types of diabetes; acute complications of diabetes, such as diabetic ketoacidosis or lactic acidosis; insufficient blood volume; subjects with diabetic nephropathy complications, rheumatic immune disease, pregnancy, malignant tumors, infection, foot ulcer, mental illness, thyroid dysfunction, severe liver and kidney dysfunction, anemia, heart failure, or respiratory insufficiency; mental illness, cognitive impairment, and patients lacking in self-care ability; and patients with a history of hyperuricemia or gout.

\section{Study design}

Subjects diagnosed with T2DM were randomized using a random number table to the treatment group (with dapagliflozin $10 \mathrm{mg}$; once daily in the morning) and the control group (without dapagliflozin). Increased doses of background antidiabetic agents in the control group were employed to achieve and maintain glycemic targets. Background antidiabetic agents included metformin, insulin, and glycosidase inhibitors. During the qualification period, medical records were accessed for baseline information including age, sex, body mass index (BMI), and smoking using a pro forma. Parameters such as fasting plasma glucose (FPG), 2-hour postprandial plasma glucose (PPG), HbA1c, duration of T2DM, and SUA levels prior to enrollment were also recorded. For patients eligible for this study, 24-hour urine volume, 24-hour urine glucose, and 24-hour urinary uric acid were measured 2 days after admission. The above laboratory indices were repeated 3 days after achieving good glycemic control during hospitalization. During the follow-up period, patients with hyperlipidemia were asked to avoid atorvastatin, fenofibrate, and other similar drugs, and patients with hypertension were asked to avoid amlodipine besylate and losartan. For eligible subjects who were at high risk for cardiovascular and cerebrovascular diseases, clopidogrel was administered after consultation with the Department of Cardiology or Neurology as necessary. Alcohol and fructose-containing drinks were strictly forbidden. Diet was based on the standard meal for T2DM patients provided by the hospital.

The primary outcome was SUA level changes from baseline to good glycemic control. The targets of blood glucose control were as follows: FPG is $4-7 \mathrm{mmol} / \mathrm{L}, \mathrm{PPG}$ is 
6-10 mmol/L, and no serious hypoglycemic events occurring during the study period. Additional outcomes included correlations between baseline SUA levels, urinary parameters, and the changes in SUA levels.

All clinical laboratory assessments for outcomes and safety parameters were determined using standardized methods by Tianjin 4th Center Hospital. This trial was approved by the institutional review board of Tianjin 4th Center Hospital and was conducted according to the Declaration of Helsinki. Before enrollment, all the patients meeting our study criterion signed informed consent forms (Trial registration code: ChiCTR1800015830).

\section{Statistical analyses}

All the analyses were performed using the SAS 9.4 software (SAS Institute Inc., Cary, NC, USA). $P<0.05$ was considered to suggest a statistically significant difference. For continuous variables, Kolmogorov-Smirnov Z-test was conducted. Once normal distribution and homogeneity of variance were satisfied, values were expressed as the mean \pm SD and Student's $t$-test was used to compare the difference between two groups. Otherwise, values were expressed as the median and rank sum test was used. For categorical variables, chi-squared test was performed. Paired Student's $t$-test was used to explore the within-group difference in SUA levels, 24-hour urine volume, 24-hour urine glucose, and 24-hour urinary uric acid before and after intervention. An analysis of covariance model with an intervention group as an effect and baseline SUA levels as a covariate was performed. We further used linear regression and Pearson correlation analysis to test relationship between changes in SUA levels and baseline SUA levels, changes in 24-hour urine volume, 24-hour urine glucose, or 24-hour urinary uric acid.

\section{Results}

Of 59 hospitalized participants who enrolled and completed the study, 29 were assigned to receive dapagliflozin $10 \mathrm{mg}$ and continued the regimen throughout the whole study period (Figure 1). No patients were lost to follow-up. Demographic and baseline characteristics including age, sex, BMI, duration

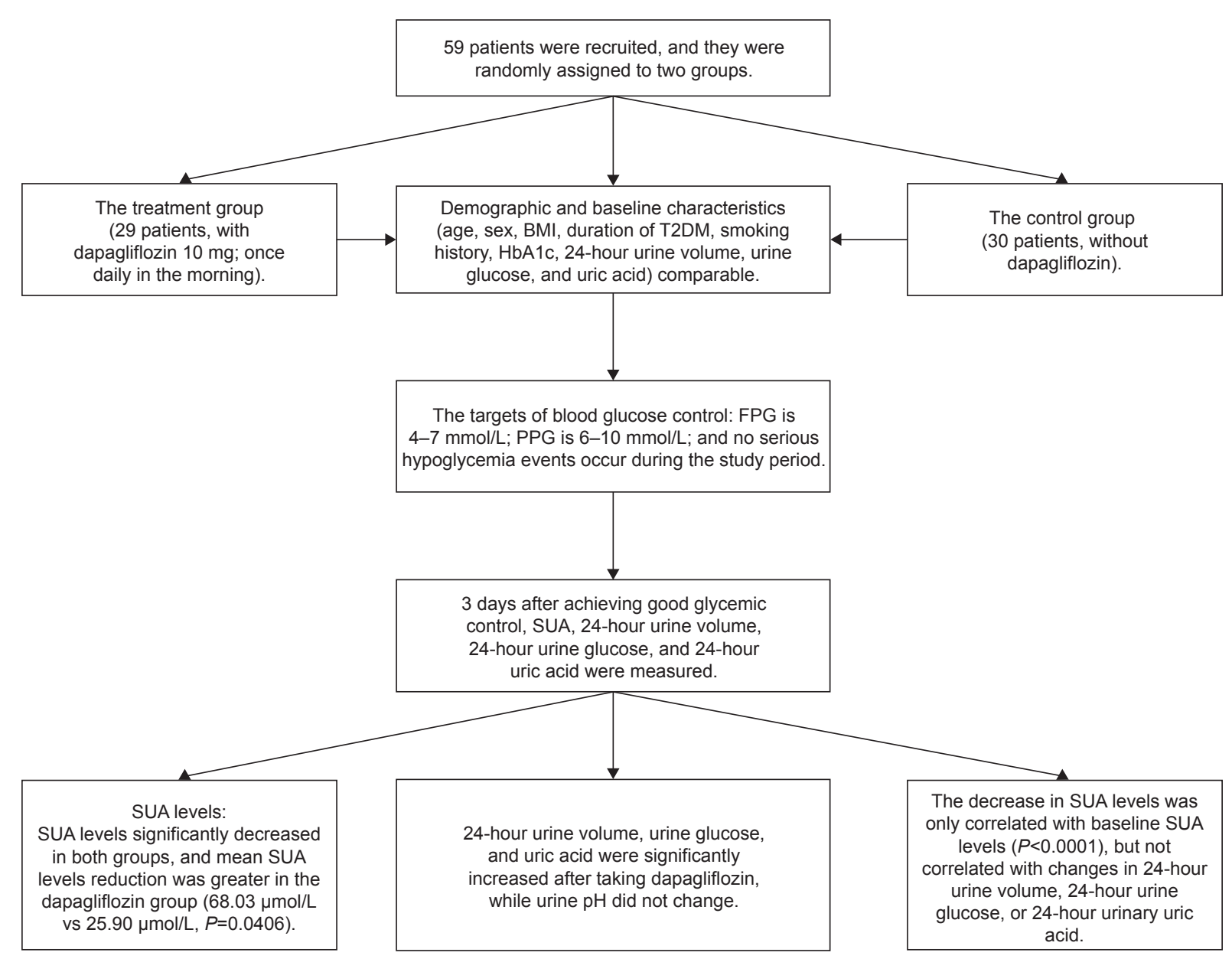

Figure I The clinical trial flow chart.

Abbreviations: BMI, body mass index; FPG, fasting plasma glucose; HbAlc, glycosylated hemoglobin; PPG, postprandial plasma glucose; SUA, serum uric acid; T2DM, type 2 diabetes mellitus. 
Table I Demographic and baseline characteristics

\begin{tabular}{|c|c|c|c|}
\hline Characteristics & Dapagliflozin $10 \mathrm{mg}(\mathrm{n}=29)$ & Control $(n=30)$ & $P$-value \\
\hline Age (years), mean $\pm S D$ & $57.77 \pm 12.29$ & $58.97 \pm 10.50$ & 0.6890 \\
\hline Men, n (\%) & $20(66.67)$ & $17(58.62)$ & 0.5960 \\
\hline Family history of T2DM, n (\%) & $21(70.00)$ & $15(51.72)$ & 0.1502 \\
\hline Smoking, n (\%) & $12(40.00)$ & 14 (48.28) & 0.5221 \\
\hline Drinking, n (\%) & $5(16.67)$ & $5(17.24)$ & 1.0000 \\
\hline Duration of T2DM (years), mean \pm SD & $12.20 \pm 6.59$ & $9.47 \pm 5.88$ & 0.0988 \\
\hline $\mathrm{BMI}\left(\mathrm{kg} / \mathrm{m}^{2}\right)$, mean $\pm \mathrm{SD}$ & $27.34 \pm 3.88$ & $25.90 \pm 3.51$ & 0.1421 \\
\hline SUA $(\mu \mathrm{mol} / \mathrm{L})$, mean $\pm \mathrm{SD}$ & $348.33 \pm 102.15$ & $326.21 \pm 103.39$ & 0.4260 \\
\hline $\mathrm{HbAlc}(\%)$, mean $\pm \mathrm{SD}$ & $9.89 \pm 1.24$ & $10.14 \pm 1.73$ & 0.8496 \\
\hline Postprandial plasma glucose (mmol/L), mean \pm SD & $15.45 \pm 2.85$ & $|5.1| \pm 2.46$ & 0.6195 \\
\hline Fasting plasma glucose $(\mathrm{mmol} / \mathrm{L})$, mean $\pm \mathrm{SD}$ & $10.54 \pm 2.22$ & $10.50 \pm 2.37$ & 0.7558 \\
\hline 24-hour urine volume $(\mathrm{L})$, mean $\pm \mathrm{SD}$ & $2.48 \pm 0.67$ & $2.67 \pm 0.84$ & 0.3772 \\
\hline 24-hour urinary uric acid $(\mu \mathrm{mol})$, mean \pm SD & $3,731.23 \pm 1,408.97$ & $3,649.91 \pm 1,075.75$ & 0.8046 \\
\hline 24 -hour urine glucose $(\mu \mathrm{mol})$, mean $\pm S D$ & $92.65 \pm 93.65$ & $80.36 \pm 131.92$ & 0.3349 \\
\hline
\end{tabular}

Abbreviations: BMI, body mass index; HbAlc, glycosylated hemoglobin; SUA, serum uric acid; T2DM, type 2 diabetes mellitus.

of T2DM, smoking history, HbA1c, 24-hour urine volume, 24-hour urine glucose, and 24-hour urinary uric acid were comparable between the two groups (Table 1). Average age was 57.77 years in the dapagliflozin group and 58.97 years in the control group $(P=0.6890)$. The duration of T2DM was not significantly longer in the dapagliflozin group (12.20 years vs 9.47 years; $P=0.0988)$. Baseline SUA levels were similar between the two groups $(348.33 \pm 102.15$ vs $326.21 \pm 103.39$; $P=0.4260)$.

At the end of the study when all participants achieved good glycemic control, FPG, PPG, and SUA levels significantly decreased in both groups (Table 2). In the dapagliflozin group, mean SUA levels dropped from 348.33 to $280.30 \mu \mathrm{mol} / \mathrm{L}$ and in the control group, mean SUA levels dropped from 326.21 to $300.31 \mu \mathrm{mol} / \mathrm{L}$. Mean SUA level reduction from baseline was significantly greater for the dapagliflozin group than the control group (68.03 vs $25.90 \mu \mathrm{mol} / \mathrm{L}$ reduction; $P=0.0406$; Table 3 ). Adjusted

Table 2 Changes in FPG, PPG, and SUA levels before and after treatment in the two groups

\begin{tabular}{|c|c|c|c|}
\hline \multirow[t]{2}{*}{ Groups } & \multicolumn{2}{|l|}{ Treatment } & \multirow[t]{2}{*}{$P$-value } \\
\hline & Before & After & \\
\hline \multicolumn{4}{|l|}{ Dapagliflozin $(n=29)$} \\
\hline FPG & $10.54 \pm 2.22$ & $7.11 \pm 0.96$ & $<0.001$ \\
\hline PPG & $15.45 \pm 2.85$ & $9.77 \pm 1.21$ & $<0.001$ \\
\hline SUA & $348.33 \pm 102.15$ & $280.30 \pm 71.53$ & $<0.001$ \\
\hline \multicolumn{4}{|l|}{ Control $(n=30)$} \\
\hline FPG & $10.50 \pm 2.37$ & $6.94 \pm 0.76$ & $<0.001$ \\
\hline PPG & $15.11 \pm 2.46$ & $9.4 I \pm I .5 I$ & $<0.001$ \\
\hline SUA & $326.21 \pm 103.39$ & $300.31 \pm 91.24$ & 0.013 \\
\hline
\end{tabular}

Abbreviations: FPG, fasting plasma glucose; PPG, 2-hour postprandial plasma glucose; SUA, serum uric acid. mean SUA levels were lower in the dapagliflozin group (273.28 vs $307.57 \mu \mathrm{mol} / \mathrm{L} ; P=0.0089$; Table 4). However, the differences in mean change in FPG and PPG between groups were not statistically significant $(P=0.8318$ for FPG and $P=0.9847$ for PPG).

Table 5 indicates the changes in urinary parameters in the dapagliflozin group. 24-hour urine volume, 24-hour urine glucose, and 24-hour urinary uric acid were significantly increased after taking dapagliflozin, while urine $\mathrm{pH}$ did not change. In T2DM patients treated with dapagliflozin, the decrease in SUA levels was correlated with baseline SUA levels $(P<0.0001)$ but not correlated with changes in 24-hour urine volume, 24-hour urine glucose, or 24-hour urinary uric acid (Table 6). Simple linear regression analysis suggested that increased baseline SUA levels were associated with more

Table 3 Comparisons of changes in FPG, PPG, and SUA levels between the dapagliflozin group and the control group

\begin{tabular}{|l|l|l|l|}
\hline & $\begin{array}{l}\text { Dapagliflozin } \\
(\mathbf{n}=\mathbf{2 9})\end{array}$ & $\begin{array}{l}\text { Control } \\
\mathbf{( n = 3 0 )}\end{array}$ & P-value \\
\hline FPG (mmol/L) & & & \\
Before treatment & $10.54 \pm 2.22$ & $10.50 \pm 2.37$ & 0.7558 \\
After treatment & $7.1 \mathrm{I} \pm 0.96$ & $6.94 \pm 0.76$ & 0.4689 \\
$\Delta$ FPG & $-3.43 \pm 2.14$ & $-3.56 \pm 2.36$ & 0.8318 \\
PPG (mmol/L) & & & \\
Before treatment & $15.45 \pm 2.85$ & $15.1 \mathrm{I} \pm 2.46$ & 0.6195 \\
After treatment & $9.77 \pm 1.21$ & $9.41 \pm 1.5 \mathrm{I}$ & 0.1269 \\
$\Delta$ PPG & $-5.69 \pm 2.76$ & $-5.70 \pm 2.56$ & 0.9847 \\
SUA ( $\mu$ mol/L) & & & \\
Before treatment & $348.33 \pm 102.15$ & $326.21 \pm 103.39$ & 0.4260 \\
After treatment & $280.30 \pm 71.53$ & $300.31 \pm 91.24$ & 0.3516 \\
$\Delta S U A$ & $-68.03 \pm 66.85$ & $-25.90 \pm 52.33$ & 0.0406 \\
\hline
\end{tabular}

Abbreviations: FPG, fasting plasma glucose; PPG, 2-hour postprandial plasma glucose; SUA, serum uric acid. 
Table 4 Comparisons of adjusted mean SUA levels between the dapagliflozin group and the control group

\begin{tabular}{|l|l|l|}
\hline & $\begin{array}{l}\text { Dapagliflozin } \\
(\mathbf{n = 2 9 )}\end{array}$ & $\begin{array}{l}\text { Control } \\
(\mathbf{n = 3 0 )}\end{array}$ \\
\hline $\mathrm{N}$ & 30 & 29 \\
\hline Adjusted mean & 273.28 & 307.57 \\
\hline Standard error & 8.85 & 9.00 \\
\hline $\begin{array}{l}\text { Difference (dapagliflozin - } \\
\text { control) and 95\% Cl }\end{array}$ & \multicolumn{2}{|c|}{$34.29(8.94,59.64)$} \\
\hline P-value & \multicolumn{2}{|c|}{0.0089} \\
\hline
\end{tabular}

Abbreviation: SUA, serum uric acid.

SUA changes in the dapagliflozin group $(P<0.001 ;$ Table 7$)$ than in the control group $(P=0.010)$.

\section{Discussion}

To the best of our knowledge, the present study is the first one that is designed to examine the effects of dapagliflozin on SUA levels in hospitalized patients with inadequately controlled T2DM. The results suggest that the addition of $10 \mathrm{mg}$ dapagliflozin to treatment regimen resulted in comparable glycemic control and significantly reduced SUA levels in a hospital setting. In addition, for T2DM patients treated with dapagliflozin, the decrease in SUA levels was correlated with baseline SUA levels $(P<0.0001)$ but not with changes in 24-hour urine volume, 24-hour urine glucose, or 24-hour urinary uric acid.

Dapagliflozin is an SGLT2 inhibitor that has been reported to significantly improve glycemic control as monotherapy or as an adjunct to other antihyperglycemic drugs including metformin, insulin, or sitagliptin with acceptable tolerability and safety through an insulin-independent mechanism in several RCTs. ${ }^{21,23,25,31-34}$ Underlying mechanisms might reduce glucose reabsorption in the proximal tubule and subsequently increase glucose excretion in the urine. ${ }^{35}$ In addition to hypoglycemic effect, dapagliflozin has been shown to have other benefits, including a tendency for improved cardiovascular outcomes, total body weight loss, blood pressure reduction, and lowering of SUA levels. ${ }^{19-28}$
The present study indicated improved glycemic control after treatment with dapagliflozin, although no significant difference was found between groups.

Our study suggested additional beneficial effects of dapagliflozin on SUA reduction, which was consistent with previously published studies. ${ }^{29,30} \mathrm{~A}$ mean reduction of $68.03 \mu \mathrm{mol} / \mathrm{L}$ of SUA levels was observed in the dapagliflozin group, with only $25.90 \mu \mathrm{mol} / \mathrm{L}$ reduction in the control group. The considerable reduction of SUA levels was similar to that shown in a meta-analysis, which revealed a $67.98 \mu \mathrm{mol} / \mathrm{L}$ mean reduction in SUA levels for the dapagliflozin $10 \mathrm{mg}$ group. ${ }^{30}$ Moreover, higher dapagliflozin dose is associated with a greater SUA reduction (from 5 to $50 \mathrm{mg}$ ). In fact, to pool estimates from multiple RCTs can be challenging, as inclusion or exclusion criterion varied across these trials. For example, status was not clearly identified and included hyperuricemia, gout, or medications affecting SUA levels in majority of the included trials. These conditions were crucial for the assessment of SUA level changes induced by dapagliflozin. However, almost all studies have found a hypouricemic effect after administration of dapagliflozin, enabling reasonable combination of results from different trials.

The present study also found that decrease in SUA levels was correlated with baseline pre-intervention SUA levels. Potential mechanisms behind hypouricemic effect of dapagliflozin have not yet been established; however, the renal SLC2A9 transporter that transports both uric acid and D-glucose may be involved. ${ }^{36,37}$ SGLT2 inhibitors lead to sustained glucose loss in the urine accompanied by an increased interchange of uric acid in the lumen membrane of tubular epithelial cells, which subsequently causes increased uric acid excretion and lower SUA levels. However, this study failed to detect a correlation between changes in SUA levels and changes in 24-hour urine volume, 24-hour urine glucose, or 24-hour urinary uric acid. This nonsignificant result may be due to a small sample or short duration of follow-up. Besides, other mechanisms possibly played a role in the hypouricemic effect of dapagliflozin. Further

Table 5 Changes in urinary parameters before and after treatment in the dapagliflozin group

\begin{tabular}{|l|l|l|l|}
\hline Parameters & \multicolumn{2}{|l|}{$\begin{array}{l}\text { Dapagliflozin } \\
\text { I0 mg }\end{array}$} & \multirow{2}{*}{ P-value } \\
\cline { 2 - 3 } & Before & After & \\
\hline 24-hour urine volume $(\mathrm{L})$ & $2.48 \pm 0.67$ & $2.97 \pm 0.8 \mathrm{I}$ & $0.003 \mathrm{I}$ \\
24-hour urinary uric acid $(\mu \mathrm{mol})$ & $3,73 \mathrm{I} .2 \pm \mathrm{I}, 408.97$ & $4,454.27 \pm \mathrm{I}, 500.52$ & 0.0035 \\
24-hour urine glucose $(\mu \mathrm{mol})$ & $92.65 \pm 93.65$ & $284.68 \pm 215.60$ & $<0.000 \mathrm{I}$ \\
24-hour urine $\mathrm{pH}$ & $5.95 \pm 0.74$ & $5.88 \pm 0.6 \mathrm{I}$ & 0.5722 \\
\hline
\end{tabular}


Table 6 Pearson correlation analysis between changes in SUA levels and baseline SUA levels, changes in 24-hour urine volume, 24-hour urine glucose, or 24-hour urinary uric acid

\begin{tabular}{|l|l|l|}
\hline & $\boldsymbol{r}$ & $\boldsymbol{P}$-value \\
\hline Baseline SUA levels & 0.72 & $<0.000 \mathrm{I}$ \\
Changes in 24-hour urine volume & 0.05 & 0.7834 \\
Changes in 24-hour urine glucose & $0.1 \mathrm{I}$ & 0.5650 \\
Changes in 24-hour urinary uric acid & $-0.0 \mathrm{I}$ & 0.9784 \\
\hline
\end{tabular}

Abbreviation: SUA, serum uric acid.

trials and fundamental studies are needed to clarify such phenomena.

This is the first study specially focusing on hypouricemic effects of dapagliflozin in hospitalized patients with inadequately controlled T2DM. Hospitalized T2DM patients can be closely monitored for suitable diet and tested for various indicators. Meanwhile, good glycemic control was an important target for hospitalized T2DM patients, which may be linked to improved glycemic control after discharge. ${ }^{38,39}$ We excluded T2DM patients with renal insufficiency, a history of hyperuricemia or gout, whose SUA levels might have been altered by disease progression. The current study also has several limitations. First, except for dapagliflozin, other coadministered hypoglycemic agents potentiality affected SUA levels which may have had an influence on the results. Second, a relatively small sample size limits the generalization of the study and the conclusions need to be carefully interpreted. Third, data regarding changes in 24-hour urine volume, 24-hour urine glucose, and 24-hour urinary uric acid in the control group were not available, which resulted in failure to compare the difference in changes of these outcomes between groups. Finally, the study had a short duration of follow-up, and multiple measurements for indicators involved were not possible.

\section{Conclusion}

Dapagliflozin was shown to improve glycemic control and lower SUA levels in hospitalized T2DM patients with inadequate glycemic control. Given adverse effects associated with hyperuricemia, dapagliflozin was considered to provide additional benefits for hospitalized T2DM patients over other oral antidiabetic agents. The hypouricemic effect may not be attributable to the increase in the urinary excretion

Table 7 Linear regression between changes in SUA levels and baseline SUA levels

\begin{tabular}{|l|l|l|l|}
\hline Group & $\boldsymbol{\beta}$ & $\boldsymbol{R}^{2}$ & $\boldsymbol{P}$-value \\
\hline Dapagliflozin $(\mathrm{n}=29)$ & 0.469 & 0.513 & $<0.001$ \\
Control $(\mathrm{n}=30)$ & 0.239 & 0.222 & 0.010 \\
\hline
\end{tabular}

Abbreviation: SUA, serum uric acid. rate of uric acid. Longer-time trials are required to further illuminate the hypouricemic effect of dapagliflozin and its corresponding mechanisms.

\section{Acknowledgments}

This work was supported by the High-Priority Health Projects of Tianjin (16KG146); Tianjin Major Science and Technology Projects (17ZXMFSY00200). Zhaohu Hao and Xiao Huang are co-first authors for this study.

\section{Disclosure}

The authors report no conflicts of interest in this work.

\section{References}

1. Grassi D, Ferri L, Desideri G, et al. Chronic hyperuricemia, uric acid deposit and cardiovascular risk. Curr Pharm Des. 2013;19(13): 2432-2438.

2. Bobulescu IA, Moe OW. Renal transport of uric acid: evolving concepts and uncertainties. Adv Chronic Kidney Dis. 2012;19(6):358-371.

3. Mutluay R, Deger SM, Bahadir E, Durmaz AO, Citil R, Sindel S. Uric acid is an important predictor for hypertensive early atherosclerosis. Adv Ther. 2012;29(3):276-286.

4. Fukui M, Tanaka M, Shiraishi E, et al. Serum uric acid is associated with microalbuminuria and subclinical atherosclerosis in men with type 2 diabetes mellitus. Metabolism. 2008;57(5):625-629.

5. Loeffler LF, Navas-Acien A, Brady TM, Miller ER 3rd, Fadrowski JJ. Uric acid level and elevated blood pressure in US adolescents: National Health and Nutrition Examination Survey, 1999-2006. Hypertension. 2012;59(4):811-817.

6. Kim SY, Guevara JP, Kim KM, Choi HK, Heitjan DF, Albert DA. Hyperuricemia and coronary heart disease: a systematic review and meta-analysis. Arthritis Care Res (Hoboken). 2010;62(2): $170-180$.

7. Han T, Meng X, Shan R, et al. Temporal relationship between hyperuricemia and obesity, and its association with future risk of type 2 diabetes. Int J Obes (Lond). 2018;42(7):1336-1344.

8. Bhole V, Choi JW, Kim SW, de vera M, Choi H. Serum uric acid levels and the risk of type 2 diabetes: a prospective study. Am J Med. 2010; 123(10):957-961.

9. Chien KL, Chen MF, Hsu HC, et al. Plasma uric acid and the risk of type 2 diabetes in a Chinese community. Clin Chem. 2008;54(2): $310-316$.

10. Krishnan E, Pandya BJ, Chung L, Hariri A, Dabbous O. Hyperuricemia in young adults and risk of insulin resistance, prediabetes, and diabetes: a 15-year follow-up study. Am J Epidemiol. 2012;176(2):108-116.

11. Juraschek SP, McAdams-Demarco M, Miller ER, et al. Temporal relationship between uric acid concentration and risk of diabetes in a community-based study population. Am J Epidemiol. 2014;179(6): 684-691.

12. Madianov IV, Balabolkin MI, Markov DS, Markova TN. Main causes of hyperuricemia in diabetes mellitus. Ter Arkh. 2000;72(2):55-58.

13. Yu S, Chen Y, Hou X, et al. Serum uric acid levels and diabetic peripheral neuropathy in type 2 diabetes: a systematic review and meta-analysis. Mol Neurobiol. 2016;53(2):1045-1051.

14. Deng Z, Gu Y, Hou X, et al. Association between uric acid, cancer incidence and mortality in patients with type 2 diabetes: Shanghai diabetes registry study. Diabetes Metab Res Rev. 2016;32(3):325-332.

15. Valbusa F, Bertolini L, Bonapace S, et al. Relation of elevated serum uric acid levels to incidence of atrial fibrillation in patients with type 2 diabetes mellitus. Am J Cardiol. 2013;112(4):499-504.

16. Li Q, Yang Z, Lu B, et al. Serum uric acid level and its association with metabolic syndrome and carotid atherosclerosis in patients with type 2 diabetes. Cardiovasc Diabetol. 2011;10:72. 
17. Kalra S. Sodium glucose co-transporter-2 (SGLT2) inhibitors: a review of their basic and clinical pharmacology. Diabetes Ther. 2014; 5(2):355-366.

18. Abdul-Ghani MA, DeFronzo RA. Dapagliflozin for the treatment of type 2 diabetes. Expert Opin Pharmacother. 2013;14(12): 1695-1703.

19. Strojek K, Yoon KH, Hruba V, Elze M, Langkilde AM, Parikh S. Effect of dapagliflozin in patients with type 2 diabetes who have inadequate glycaemic control with glimepiride: a randomized, 24-week, doubleblind, placebo-controlled trial. Diabetes Obes Metab. 2011;13(10): 928-938.

20. Ferrannini E, Ramos SJ, Salsali A, Tang W, List JF. Dapagliflozin monotherapy in type 2 diabetic patients with inadequate glycemic control by diet and exercise: a randomized, double-blind, placebocontrolled, phase 3 trial. Diabetes Care. 2010;33(10):2217-2224.

21. Bailey CJ, Gross JL, Pieters A, Bastien A, List JF. Effect of dapagliflozin in patients with type 2 diabetes who have inadequate glycaemic control with metformin: a randomised, double-blind, placebo-controlled trial Lancet. 2010;375(9733):2223-2233.

22. Kirk R. Diabetes: efficacy of dapagliflozin associated with renal function. Nat Rev Endocrinol. 2013;9(12):688

23. Wilding JP, Woo V, Soler NG, et al. Long-term efficacy of dapagliflozin in patients with type 2 diabetes mellitus receiving high doses of insulin: a randomized trial. Ann Intern Med. 2012;156(6):405-415.

24. Nauck MA, Del Prato S, Meier JJ, et al. Dapagliflozin versus glipizide as add-on therapy in patients with type 2 diabetes who have inadequate glycemic control with metformin: a randomized, 52-week, doubleblind, active-controlled noninferiority trial. Diabetes Care. 2011;34(9): 2015-2022.

25. Bailey CJ, Gross JL, Hennicken D, Iqbal N, Mansfield TA, List JF. Dapagliflozin add-on to metformin in type 2 diabetes inadequately controlled with metformin: a randomized, double-blind, placebo-controlled 102-week trial. BMC Med. 2013;11:43.

26. Jabbour SA, Hardy E, Sugg J, Parikh S; Study 10 Group. Dapagliflozin is effective as add-on therapy to sitagliptin with or without metformin: a 24-week, multicenter, randomized, double-blind, placebo-controlled study. Diabetes Care. 2014;37(3):740-750.

27. List JF, Woo V, Morales E, Tang W, Fiedorek FT. Sodium-glucose cotransport inhibition with dapagliflozin in type 2 diabetes. Diabetes Care. 2009;32(4):650-657.
28. Kato K, Suzuki K, Aoki C, et al. The effects of intermittent use of the SGLT-2 inhibitor, dapagliflozin, in overweight patients with type 2 diabetes in Japan: a randomized, crossover, controlled clinical trial. Expert Opin Pharmacother. 2017;18(8):743-751.

29. Ahmadieh H, Azar S. Effects of sodium glucose cotransporter-2 inhibitors on serum uric acid in type 2 diabetes mellitus. Diabetes Technol Ther. 2017;19(9):507-512.

30. Zhao Y, Xu L, Tian D, et al. Effects of sodium-glucose co-transporter 2 (SGLT2) inhibitors on serum uric acid level: a meta-analysis of randomized controlled trials. Diabetes Obes Metab. 2018;20(2):458-462.

31. Scorsone A, Saura G, Fleres M, et al. Efficacy and renal safety of dapagliflozin in patients with type 2 diabetes mellitus also receiving metformin: a real-life experience. J Diabetes Res. 2018;8501418, 5 pages.

32. Schumm-Draeger PM, Burgess L, Korányi L, Hruba V, HamerMaansson JE, de Bruin TW. Twice-daily dapagliflozin co-administered with metformin in type 2 diabetes: a 16-week randomized, placebocontrolled clinical trial. Diabetes Obes Metab. 2015;17(1):42-51.

33. Wu B, Zheng H, Gu J, et al. Effects of sodium-glucose cotransporter 2 inhibitors in addition to insulin therapy on cardiovascular risk factors in type 2 diabetes patients: a meta-analysis of randomized controlled trials. J Diabetes Investig. Epub 2018 Jun 19.

34. Araki E, Onishi Y, Asano M, et al. Efficacy and safety of dapagliflozin in addition to insulin therapy in Japanese patients with type 2 diabetes: results of the interim analysis of 16-week double-blind treatment period. $J$ Diabetes Investig. 2016;7(4):555-564.

35. Chino Y, Samukawa Y, Sakai S, et al. SGLT2 inhibitor lowers serum uric acid through alteration of uric acid transport activity in renal tubule by increased glycosuria. Biopharm Drug Dispos. 2014;35(7):391-404.

36. McGill JB. The SGLT2 inhibitor empagliflozin for the treatment of type 2 diabetes mellitus: a bench to bedside review. Diabetes Ther. 2014; 5(1):43-63.

37. Li S, Sanna S, Maschio A, et al. The GLUT9 gene is associated with serum uric acid levels in Sardinia and Chianti cohorts. PLoS Genet. 2007;3(11):e194.

38. Cook CB, Elias B, Kongable GL, Potter DJ, Shepherd KM, McMahon D. Diabetes and hyperglycemia quality improvement efforts in hospitals in the United States: current status, practice variation, and barriers to implementation. Endocr Pract. 2010;16(2):219-230.

39. Clement S, Braithwaite SS, Magee MF, et al. Management of diabetes and hyperglycemia in hospitals. Diabetes Care. 2004;27(2):553-591.
Therapeutics and Clinical Risk Management

\section{Publish your work in this journal}

Therapeutics and Clinical Risk Management is an international, peerreviewed journal of clinical therapeutics and risk management, focusing on concise rapid reporting of clinical studies in all therapeutic areas outcomes, safety, and programs for the effective, safe, and sustained use of medicines. This journal is indexed on PubMed Central, CAS,

\section{Dovepress}

EMBase, Scopus and the Elsevier Bibliographic databases. The manuscript management system is completely online and includes a very quick and fair peer-review system, which is all easy to use. Visit http://www.dovepress.com/testimonials.php to read real quotes from published authors. 